\title{
The Equivalent Linearization Method with a Weighted Averaging for Solving Undamped Nonlinear Oscillators
}

\author{
D. V. Hieu $\mathbb{D}^{1},{ }^{1}$ N. Q. Hai, ${ }^{2}$ and D. T. Hung ${ }^{1}$ \\ ${ }^{1}$ Thai Nguyen University of Technology, Thai Nguyen, Vietnam \\ ${ }^{2}$ Hanoi Architectural University, Ha Noi, Vietnam \\ Correspondence should be addressed to D. V. Hieu; hieudv@tnut.edu.vn
}

Received 27 June 2017; Accepted 5 March 2018; Published 18 April 2018

Academic Editor: Peter G. L. Leach

Copyright (c) 2018 D. V. Hieu et al. This is an open access article distributed under the Creative Commons Attribution License, which permits unrestricted use, distribution, and reproduction in any medium, provided the original work is properly cited.

\begin{abstract}
The Equivalent Linearization Method (ELM) with a weighted averaging is applied to analyze five undamped oscillator systems with nonlinearities. The results obtained via this method are compared with the ones achieved by Parameterized Perturbation Method (PPM), Min-Max Approach (MMA), Variational Iteration Method (VIM), Homotopy Perturbation Method (HPM), Energy Balance Method (EBM), Harmonic Balance Method (HBM), 4th-Order Runge-Kutta Method, and the exact ones. The obtained results demonstrate that this method is very convenient for solving nonlinear equations and also can be successfully applied to a lot of practical engineering and physical problems.
\end{abstract}

\section{Introduction}

Nonlinear oscillation problem is very important in the physical science, mechanical structures, and other kinds of mathematical sciences. Most of real systems are modeled by nonlinear differential equations which are important issues in mechanical structures, mathematical physics, and engineering. In most cases, it is difficult to solve such equations, especially analytically; and in addition, the most important information, such as the natural circular frequency of a nonlinear oscillation which depends on the initial conditions, will be lost during the procedure of numerical simulation.

Recently, nonlinear oscillator models have been widely considered in physics and engineering. It is obvious that there are many nonlinear equations in the study of different branches of science which do not have analytical solutions. Due to the limitation of existing exact solutions, many analytical approaches have been investigated. Many researchers have been working on various analytical methods for solving nonlinear oscillation systems in the last decades, such as Homotopy Perturbation Method (HPM) [15], Max-Min Approach (MMA) [5-10], Variational Iteration Method (VIM) [2, 5], Energy Balance Method (EBM) [5, 1116], Amplitude-Frequency Formulation (AFF) $[5-7,15,17$, 18], Improved Amplitude-Frequency Formulation (IAFF) [5],
Parameter Expansion Method (PEM) [5, 7, 18-20], Homotopy Analysis Method (HAM) [5, 21], Modified Homotopy Perturbation Method (MHPM) [5, 6], Modified LindstedtPoincare Method [22], Harmonic Balance Method [23, 24], and combined Newton's Method with the Harmonic Balance Method [25].

The Equivalent Linearization Method is one of the common approaches to approximate analysis of dynamical systems. The original linearization for deterministic systems was proposed by Krylov and Bogoliuboff [26]. Then Caughey expanded the method for stochastic systems [27]. Thenceforward, there have been some extended versions of the Equivalent Linearization Method [28-32]. It has been shown that the Equivalent Linearization Method is presently the simplest tool widely used for analyzing nonlinear stochastic problems. Nevertheless, the accuracy of the Equivalent Linearization Method with conventional averaging normally reduces for middle or strong nonlinear systems. A reason is that some terms will vanish in the averaging process; for example, the averaging value of the functions $\sin (t)$ and $\cos (t)$ over one period is equal to zero. Recently, Anh proposed a new way for determining averaging values: instead of using conventional averaging process the author introduced weighted coefficient functions $h(t)$ [31]; by this manner, the averaging value is calculated in a new way called the weighted averaging value. 
This proposed method has been applied effectively to analyze some strongly nonlinear oscillations such as the nonlinear Duffing oscillator with third, fifth, and seventh powers of the amplitude, the strongly nonlinear oscillators in forms $\left(1+\varepsilon u^{2}\right) \ddot{u}+u=0$ and $\ddot{u}+u^{3} /\left(1+u^{2}\right)=0$, and the cubic Duffing with discontinuity [33].

In this paper, the Equivalent Linearization Method with a weighted averaging continues to be applied to analyze five undamped nonlinear oscillators: the Duffing oscillator with cubic nonlinearity (Example 1); the motion of simple pendulum attached to a rotating rigid frame (Example 2); the motion of a mass attached to the center of a stretched elastic wire (Example 3); the Duffing oscillator with discontinuity (Example 4); and the nonlinear oscillator with fractional elastic force (Example 5). It should be emphasized that the generalized forms of the Duffing equation (in Example 1) and the oscillator (in Example 3) have been solved by Energy Balance Method [12, 16], and the generalized form of the nonlinear oscillator with fractional elastic force (in Example 5) has been solved by Energy Balance Method and Variational Approach [14]; these solutions are achieved by Younesian et al. [12, 14, 16]. The frequency-amplitude relationship is analytically obtained by using this proposed method. Accuracy and validity of results are then presented by comparing the results with the ones obtained by the other well-known techniques and the exact and numerical ones.

\section{The Equivalent Linearization Method with a Weighted Averaging}

2.1. The Equivalent Linearization Method. In order to introduce the general idea of the Equivalent Linearization Method, we consider a nonlinear oscillator governed by the following equation [33]:

$$
\ddot{X}+2 h \dot{X}+\omega_{0}^{2} X+g(\dot{X}, X)=0,
$$

where $g(\dot{X}, X)$ is a nonlinear function only depending on two variables of velocity $\dot{X}(t)$ and displacement $X(t)$ and $h$ and $\omega_{0}$ are constants. The equivalent linear oscillator is described by the equation as follows:

$$
\ddot{X}+(2 h+\mu) \dot{X}+\left(\omega_{0}^{2}+\lambda\right) X=0 \text {. }
$$

The equation error between the two oscillators is taken as

$$
e(\dot{X}, X)=g(\dot{X}, X)-\mu \dot{X}-\lambda X
$$

The coefficients of linearization in the linearized equation (2) are found from an optimal criterion. There are some criteria for determining these coefficients. The most common criterion is the mean square error criterion which requires that the mean square of equation error be minimum:

$$
\left\langle e^{2}(\dot{X}, X)\right\rangle=\left\langle(g(\dot{X}, X)-\mu \dot{X}-\lambda X)^{2}\right\rangle \rightarrow \min _{\mu, \lambda}
$$

Thus,

$$
\begin{aligned}
& \frac{\partial}{\partial \lambda}\left\langle e^{2}(\dot{X}, X)\right\rangle=0 \\
& \frac{\partial}{\partial \mu}\left\langle e^{2}(\dot{X}, X)\right\rangle=0
\end{aligned}
$$

yields

$$
\begin{aligned}
& \lambda=\frac{\langle g X\rangle\left\langle\dot{X}^{2}\right\rangle-\langle g \dot{X}\rangle\langle X \dot{X}\rangle}{\left\langle X^{2}\right\rangle\left\langle\dot{X}^{2}\right\rangle-\langle X \dot{X}\rangle^{2}} \\
& \mu=\frac{\langle g \dot{X}\rangle\left\langle X^{2}\right\rangle-\langle g X\rangle\langle X \dot{X}\rangle}{\left\langle X^{2}\right\rangle\left\langle\dot{X}^{2}\right\rangle-\langle X \dot{X}\rangle^{2}} .
\end{aligned}
$$

In the formulations in (4), (6a), and (6b), the symbol $\langle\bullet\rangle$ denotes the time-averaging operator in classical meaning [26]:

$$
\langle f(t)\rangle=\lim _{T \rightarrow+\infty} \frac{1}{T} \int_{0}^{T} f(t) d t .
$$

For a $\omega$-frequency function $f(\omega t)$, the averaging process is taken during one period $T$; that is,

$$
\langle f(\omega t)\rangle=\frac{1}{T} \int_{0}^{T} f(\omega t) d t=\frac{1}{2 \pi} \int_{0}^{2 \pi} f(\tau) d \tau,
$$

$$
\tau=\omega t \text {. }
$$

In this technique, the importance of the attended terms is considered to be the same on time scale. In fact, their roles generally differ from time to time. That may be one of the reasons causing the classical equivalent replacement to be effective only for oscillators with weak nonlinearity but normally not being good for ones with strong nonlinearity. In order to improve this shortcoming, the averaging operation with weighting functions is proposed as in the next section. This idea is introduced by Anh [31].

2.2. The Weighted Averaging. The conventional averaging value of an integrable deterministic function $x(t)$ on a domain $D:(0, d)$ is a constant value defined by

$$
\langle x(t)\rangle=\frac{1}{d} \int_{0}^{d} x(t) d t .
$$

In many cases when the function $x(\omega t)$ is periodic with period $2 \pi / \omega$, the value $d$ is taken as $2 \pi / \omega$ and it leads to the averaging value of $x(t)$ over one period:

$$
\langle x(\omega t)\rangle=\frac{\omega}{2 \pi} \int_{0}^{2 \pi / \omega} x(\omega t) d t=\frac{1}{2 \pi} \int_{0}^{2 \pi} x(\tau) d \tau,
$$

where $\tau=\omega t$. Definition (9) has some deficiencies; for example, if (9) or (10) are equal to zero, the information about $x(t)$ will be lost. For all harmonic functions such as $\cos (n \omega t)$ and $\sin (n \omega t)$, this observation is true. In [31], Anh suggested replacing the constant coefficient $1 / d$ in (9) by a weighted coefficient function $h(t)$. Thus one gets the so-called weighted average value:

$$
\langle x(t)\rangle=\int_{0}^{d} h(t) x(t) d t
$$


with the condition

$$
\int_{0}^{d} h(t) d t=1
$$

There are three basic weighted coefficients:

(i) Basic Optimistic Weighted Coefficients. They are increasing functions of $t$ and denoted as $O(t)$. Examples are $\alpha t^{\beta}$ and $\alpha e^{\beta t}, \alpha, \beta>0$.

(ii) Basic Pessimistic Weighted Coefficients. They are decreasing functions of $t$ and denoted as $P(t)$. Examples are $\alpha t^{\beta}$ and $\alpha e^{\beta t}, \alpha<0, \beta>0$; or $\alpha>0, \beta<0$.

(iii) Neutral Weighted Coefficients. They are denoted as $N(t)$ and are constants.

An arbitrary weighted coefficient $h(t)$ can be obtained as summation and/or product of basic weighted coefficients. Example is

$$
h(t)=\sum_{i=1}^{n} A_{i} O_{i}(t)+B_{i} P_{i}(t)+C_{i} O_{i}(t) P_{i}(t)+N(t),
$$

where $A_{i}, B_{i}, C_{i}$ are constant.

In this paper, a special form of weighted coefficient is introduced as follows $[31,33]$ :

$$
h(t)=s^{2} \omega^{2} t e^{-s \omega t}, \quad s>0,
$$

where $s$ is constant.

The properties of the weighted function $h(t)$ in (14) can be viewed in $[31,33]$.

Based on the weighted coefficient (14), a new weighted averaging value is proposed:

$$
\begin{aligned}
\langle x(\omega t)\rangle & =\int_{0}^{\infty} s^{2} \omega^{2} t e^{-s \omega t} x(\omega t) d t \\
& =\int_{0}^{\infty} s^{2} \tau e^{-s \tau} x(\tau) d \tau
\end{aligned}
$$

which is a linear operator. From Laplace transform, we get, for example,

$$
\begin{aligned}
\langle\cos (n \omega t)\rangle & =\int_{0}^{\infty} s^{2} \omega^{2} t e^{-s \omega t} \cos (n \omega t) d t \\
& =\int_{0}^{\infty} s^{2} \tau e^{-s \tau} \cos (n \tau) d \tau=s^{2} \frac{s^{2}-n^{2}}{\left(s^{2}+n^{2}\right)^{2}} \\
\langle\sin (n \omega t)\rangle & =\int_{0}^{\infty} s^{2} \omega^{2} t e^{-s \omega t} \sin (n \omega t) d t \\
& =\int_{0}^{\infty} s^{2} \tau e^{-s \tau} \sin (n \tau) d \tau=s^{2} \frac{2 s n}{\left(s^{2}+n^{2}\right)^{2}} .
\end{aligned}
$$

As $\omega$-periodic functions $x(\omega t)$ can be expanded into Fourier series; hence we can easily calculate (15) by using (16).

In this paper, for the sake of comparison, the parameter $s$ is chosen equal to 2 . In the next section, some practical examples for some strongly nonlinear vibration systems are illustrated to show the applicability, accuracy, and effectiveness of the present method.

\section{Some Examples and Discussions}

3.1. Example 1. Consider the following Duffing equation with cubic nonlinearity:

$$
\ddot{u}+\alpha u+\beta u^{3}=0, \quad u(0)=A, \dot{u}(0)=0 .
$$

The linearized equation of (17) is

$$
\ddot{u}+\omega^{2} u=0 .
$$

The equation error between (17) and (18) is

$$
e(u)=\alpha u+\beta u^{3}-\omega^{2} u,
$$

where $\omega^{2}$ is determined by using the mean square criterion, as follows:

$$
\omega^{2}=\frac{\alpha\left\langle u^{2}\right\rangle+\beta\left\langle u^{4}\right\rangle}{\left\langle u^{2}\right\rangle} .
$$

The periodic solution of the linearized equation (18) is

$$
u(t)=A \cos (\omega t) \text {. }
$$

Using definition (11) and weighted coefficient (14), we can calculate averaging operators in (20):

$$
\begin{aligned}
\left\langle u^{2}\right\rangle & =\left\langle A^{2} \cos ^{2} \omega t\right\rangle \\
& =\int_{0}^{+\infty} A^{2} s^{2} \omega^{2} t e^{-s \omega t} \cos ^{2}(\omega t) d t \\
& =\int_{0}^{+\infty} A^{2} s^{2} \tau e^{-s \tau} \cos ^{2}(\tau) d \tau=A^{2} \frac{s^{4}+2 s^{2}+8}{\left(s^{2}+4\right)^{2}} \\
\left\langle u^{4}\right\rangle & =\left\langle A^{4} \cos ^{4} \omega t\right\rangle \\
& =\int_{0}^{+\infty} A^{4} s^{2} \omega^{2} t e^{-s \omega t} \cos ^{4}(\omega t) d t \\
& =\int_{0}^{+\infty} A^{4} s^{2} \tau e^{-s \tau} \cos ^{4}(\tau) d \tau \\
& =A^{4} \frac{248 s^{4}+416 s^{2}+1536+28 s^{6}+s^{8}}{\left(s^{2}+4\right)^{2}\left(s^{2}+16\right)^{2}} .
\end{aligned}
$$

Substituting (22) into (20), we get the approximate frequency of this oscillator:

$$
\omega=\sqrt{\alpha+\beta A^{2} \frac{248 s^{4}+416 s^{2}+1536+28 s^{6}+s^{8}}{\left(s^{4}+2 s^{2}+8\right)\left(s^{2}+16\right)^{2}}} .
$$

With $s$ chosen equal to 2 , we have

$$
\omega=\sqrt{\alpha+\frac{9216}{12800} \beta A^{2}}=\sqrt{\alpha+0.72 \beta A^{2}} .
$$

From (21), the approximate solution of this oscillator is

$$
u(t)=A \cos \left(\sqrt{\alpha+0.72 \beta A^{2}} t\right) .
$$

Accuracy of this method for this example is shown in Table 1 and Figures 1 and 2. Table 1 shows the comparison between the approximate frequencies $\omega_{\text {present }}$ obtained by this method, the ones obtained by Parameterized Perturbation 
Method $\omega_{\mathrm{PPM}}(\mathrm{PPM})[5]$, and the exact ones $\omega_{e}$; the relative error is again obtained for small and large values of the oscillation amplitude $A$. Table 1 shows that the maximum relative error is less than 0.4733 for this method and 2.2135 for Parameterized Perturbation Method. The numerical results obtained by three different methods are illustrated in Figures 1 and 2. Numerical results validate again accuracy of this method. As it is shown in Figures 3 and 4, validity of the solution technique is guaranteed for stronger nonlinearities. Relative error versus the oscillation amplitude is illustrated in Figure 3.

The approximate frequency obtained by PPM [5] is the same as that obtained by EMB [12], as follows:

$$
\omega_{\mathrm{PPM}}=\omega_{\mathrm{EBM}}=\sqrt{\alpha+\frac{3}{4} \beta A^{2}} .
$$

And the exact frequency of this oscillator is [5]

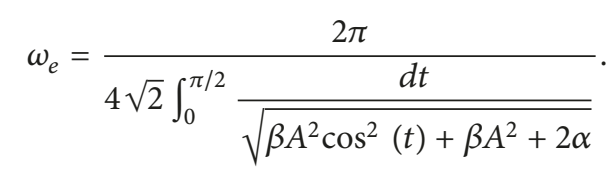

3.2. Example 2. The motion of a simple pendulum attached to a rotating rigid frame that is shown in Figure 4 has following nonlinear differential equation $[6,14]$ :

$$
\ddot{\theta}+[1-\Lambda \cos (\theta)] \sin (\theta)=0
$$

with the initial conditions:

$$
\begin{aligned}
& \theta(0)=A, \\
& \dot{\theta}(0)=0,
\end{aligned}
$$

in which $\theta$ and $t$ are generalized dimensionless displacements and time variables, and $\Lambda=\Omega^{2} r / g$.

Equation (28) can be rewritten as follows:

$$
\ddot{\theta}+\sin (\theta)-\frac{\Lambda}{2} \sin (2 \theta)=0 .
$$

$$
\begin{aligned}
& \left\langle\theta^{2}\right\rangle=\left\langle A^{2} \cos ^{2}(\omega t)\right\rangle=A^{2} \frac{s^{4}+2 s^{2}+8}{\left(s^{2}+4\right)^{2}} \\
& \left\langle\theta^{4}\right\rangle=\left\langle A^{4} \cos ^{4}(\omega t)\right\rangle=A^{2} \frac{248 s^{4}+416 s^{2}+1536+28 s^{6}+s^{8}}{\left(s^{2}+4\right)^{2}\left(s^{2}+16\right)^{2}} \\
& \left\langle\theta^{6}\right\rangle=\left\langle A^{6} \cos ^{6}(\omega t)\right\rangle=A^{6} \frac{1658880+440064 s^{2}+282496 s^{4}+45712 s^{6}+3168 s^{8}+94 s^{10}+s^{12}}{\left(s^{2}+4\right)^{2}\left(s^{2}+16\right)^{2}\left(s^{2}+36\right)^{2}} .
\end{aligned}
$$

Substituting averaging operators in (38) into (36), and then substituting (36) into (37), we get the approximate frequency (with $s$ chosen equal to 2 ):
Based on Taylor-Maclaurin expansion, the approximations of $\sin (\theta)$ and $\sin (2 \theta)$ are considered:

$$
\begin{aligned}
& \sin (\theta) \approx \theta-\frac{\theta^{3}}{6}+\frac{\theta^{5}}{120}, \\
& \sin (2 \theta) \approx 2 \theta-\frac{4 \theta^{3}}{3}+\frac{4 \theta^{5}}{15} .
\end{aligned}
$$

Substituting (31) into (30) yields

$$
\begin{aligned}
\ddot{\theta}+ & (1-\Lambda) \theta+\left(-\frac{1}{6}+\frac{2 \Lambda}{3}\right) \theta^{3}+\left(\frac{1}{120}-\frac{2 \Lambda}{15}\right) \theta^{5} \\
& =0 .
\end{aligned}
$$

The linearized equation of (32) is

$$
\ddot{\theta}+(1-\Lambda+\alpha) \theta=0 \text {. }
$$

The equation error between (32) and (33) is

$$
e(\theta)=\left(-\frac{1}{6}+\frac{2 \Lambda}{3}\right) \theta^{3}+\left(\frac{1}{120}-\frac{2 \Lambda}{15}\right) \theta^{5}-\alpha \theta .
$$

The unknown coefficient $\alpha$ is determined from the mean square error criterion:

$$
\frac{\partial}{\partial \alpha}\left\langle e^{2}(\theta)\right\rangle=0
$$

It yields

$$
\alpha=\frac{(-1 / 6+2 \Lambda / 3)\left\langle\theta^{4}\right\rangle+(1 / 120-2 \Lambda / 15)\left\langle\theta^{6}\right\rangle}{\left\langle\theta^{2}\right\rangle} .
$$

The periodic solution and the frequency of (33) are

$$
\theta(t)=A \cos (\omega t), \quad \omega=\sqrt{1-\Lambda+\alpha}
$$

Similarly to Example 1, we calculate averaging operators $\left\langle\theta^{2}\right\rangle,\left\langle\theta^{4}\right\rangle$, and $\left\langle\theta^{6}\right\rangle$ : $\omega$

$$
=\sqrt{1-\Lambda+0.72\left(-\frac{1}{6}+\frac{2 \Lambda}{3}\right) A^{2}+0.575\left(\frac{1}{120}-\frac{2 \Lambda}{15}\right) A^{4}},
$$


TABLE 1: Comparison of the approximate frequencies with the exact frequency, Example 1.

\begin{tabular}{lccccccc}
\hline$A$ & $\alpha$ & $\beta$ & $\omega_{e}$ & $\omega_{\text {PPM }}$ & R. error (\%) & $\omega_{\text {Present }}$ & R. error (\%) \\
\hline 0.1 & 0.5 & 0.1 & 0.7076 & 0.7076 & 0.0000 & 0.7076 & 0.0000 \\
0.5 & 0.1 & 2 & 0.6800 & 0.6892 & 1.3501 & 0.6782 & 0.2647 \\
1 & 2 & 0.5 & 1.5403 & 1.5411 & 0.0520 & 1.5362 & 0.2662 \\
2 & 5 & 2 & 3.2958 & 3.3166 & 0.6313 & 3.2802 & 0.4733 \\
5 & 2 & 5 & 9.5818 & 9.7852 & 2.1228 & 9.5917 & 0.1033 \\
10 & 1 & 0.5 & 6.0722 & 6.2048 & 2.0994 & 6.0828 & 0.1746 \\
15 & 0.5 & 2 & 17.9866 & 18.3848 & 2.2135 & 18.0139 & 0.1518 \\
20 & 5 & 1 & 17.0977 & 17.4642 & 2.1436 & 17.1172 & 0.1141 \\
\hline
\end{tabular}

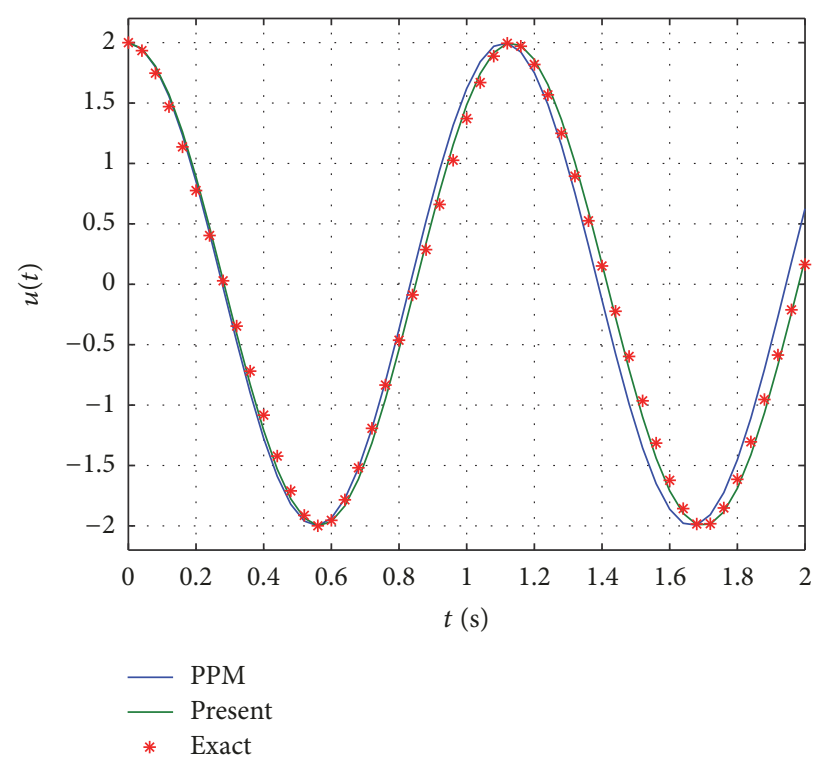

FIGURE 1: Comparison of time history diagram of displacement between the present, PPM, and exact solutions at $\alpha=2, \beta=10, A=2$, Example 1.

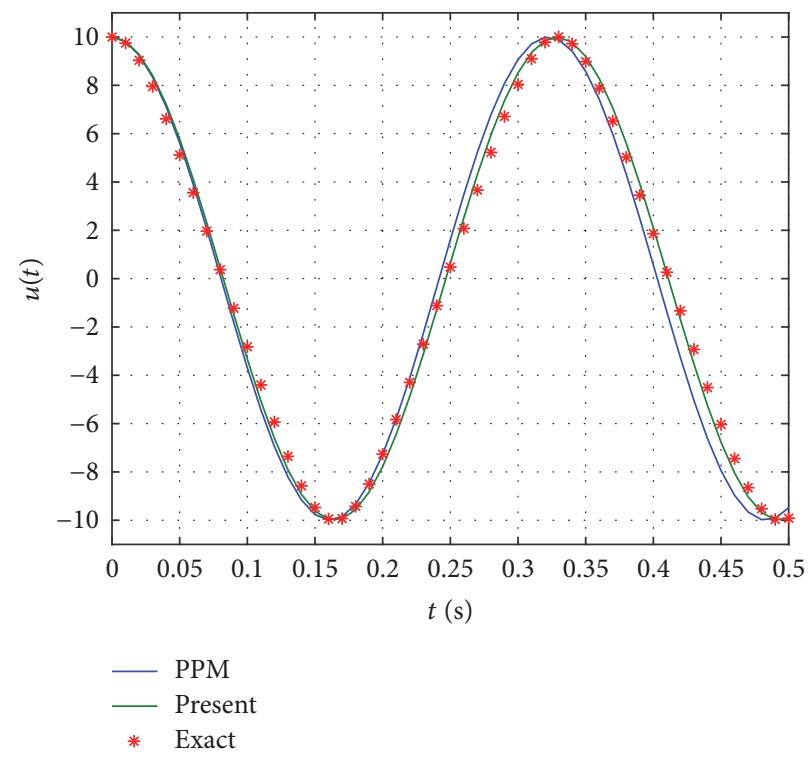

FIGURE 2: Comparison of time history diagram of displacement between the present, PPM, and exact solutions at $\alpha=5, \beta=5, A=10$, Example 1. 


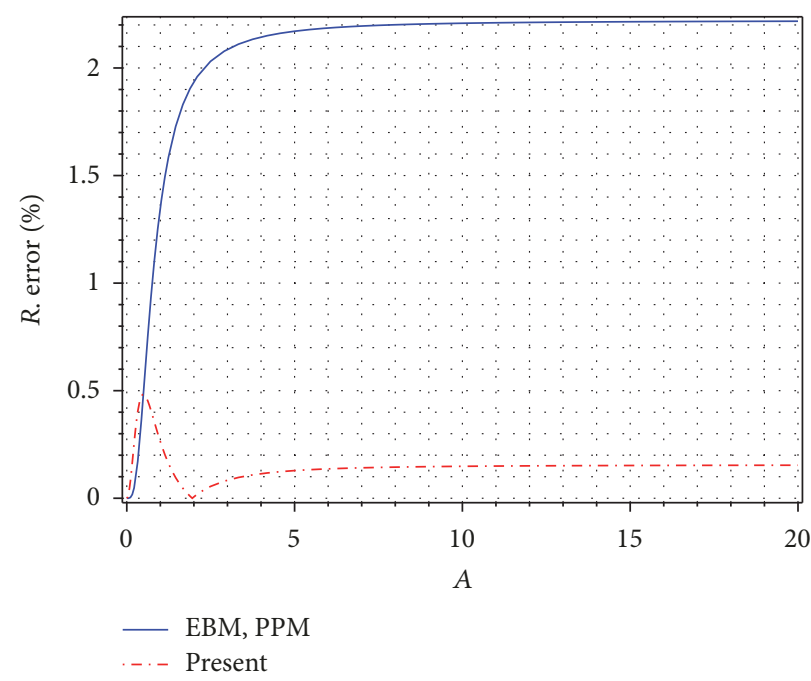

Figure 3: Comparison of relative error between the present and PPM solutions with $\alpha=2$ and $\beta=5$, Example 1 .

and the approximate solution:

$$
\theta(t)=A \cos \left[\sqrt{1-\Lambda+0.72\left(-\frac{1}{6}+\frac{2 \Lambda}{3}\right) A^{2}+0.575\left(\frac{1}{120}-\frac{2 \Lambda}{15}\right) A^{4} t}\right]
$$

Comparisons between the results of the present method and the ones achieved by Jouybari and Ramzani [6] using Min-Max Approach (MMA), by Younesian et al. [14] using EBM, and using accurate numerical method are demonstrated in Tables 2 and 3, for two cases of $\Lambda=0.75$ and $\Lambda=1$. A very interesting agreement between the results is observed.

To illustrate and verify the accuracy of the current analytical approach, the Equivalent Linearization Method with a weighted averaging, in comparison with other wellknown techniques, we performed a comparison between the results obtained by this method and outcomes achieved by Jouybari and Ramzani [6], by Younesian et al. [15], and by using the Runge-Kutta 4th-Order Method. Variations of $\theta(t)$ for different $\Lambda$ are illustrated in Figures 5-7. is

The approximate frequency obtained by using MMA [6]

$$
\omega_{\text {MMA }}=\sqrt{1-\frac{1}{8} A^{2}-\frac{1}{12} \Lambda A^{4}+\frac{1}{2} \Lambda A^{2}-\Lambda+\frac{1}{192} A^{4}} .
$$

is

The approximate frequency obtained by using EBM [15]

$$
\begin{aligned}
& \omega_{\mathrm{EBM}}=\frac{2}{A} \\
& \cdot \sqrt{\cos \left(\frac{A}{\sqrt{2}}\right)-\cos (A)-\frac{\Lambda}{4} \cos (\sqrt{2} A)+\frac{\Lambda}{4} \cos (2 A) .}
\end{aligned}
$$

3.3. Example 3. The equation of motion of a mass attached to the center of a stretched elastic wire in dimensionless form is as follows $[5,16,18]$ :

$$
\ddot{u}+u-\frac{\eta u}{\sqrt{1+u^{2}}}=0, \quad u(0)=A, \dot{u}(0)=0,
$$

where $\eta$ is a parameter, $0<\eta \leq 1$.

Equation (43) can be written as follows:

$$
\sqrt{1+u^{2}} \ddot{u}+\sqrt{1+u^{2}} u-\eta u=0 .
$$

The linearized equation of (44) is

$$
\ddot{u}+\omega^{2} u=0 .
$$

The equation error between (44) and (45) is

$$
e(u)=\sqrt{1+\varepsilon u^{2}} \ddot{u}+\left(1+\varepsilon u^{2}\right) u-\eta u-\ddot{u}-\omega^{2} u,
$$

where $\omega^{2}$ is determined by using the mean square criterion, as follows:

$$
=\frac{\left\langle\sqrt{1+u^{2}} \ddot{u} u\right\rangle+\left\langle u^{2} \sqrt{1+u^{2}}\right\rangle-\eta\left\langle u^{2}\right\rangle-\langle u \ddot{u}\rangle}{\left\langle u^{2}\right\rangle} .
$$

The periodic solution of the linearized equation (45) is

$$
u(t)=A \cos (\omega t)
$$

Using (48), we have

$$
\begin{aligned}
u \ddot{u} & =-A \cos (\omega t) A \omega^{2} \cos (\omega t)=-A^{2} \omega^{2} \cos ^{2}(\omega t) \\
& =-\omega^{2} u^{2} .
\end{aligned}
$$




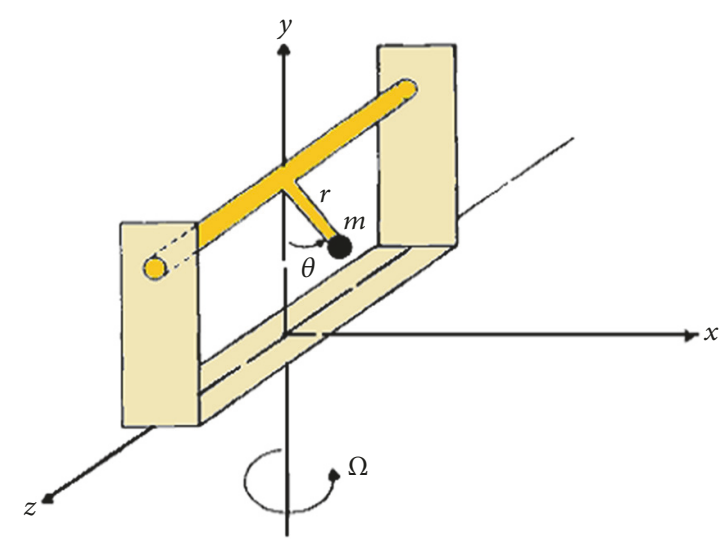

FIGURE 4: A simple pendulum attached to a rotating rigid frame.

TABLE 2: The results of present, approximate, obtained by Jouybari \& Ramzani and by Yousesian et al., and numerical solution for $\theta(t)$ when $\Lambda=0.75$ and $A=1$, Example 2 .

\begin{tabular}{|c|c|c|c|c|c|c|c|}
\hline$t$ & $\begin{array}{c}\text { Numerical } \\
\text { solution [12] }\end{array}$ & $\begin{array}{c}\text { MMA solution } \\
{[6]}\end{array}$ & $\begin{array}{c}R . \text { error } \\
(\%)\end{array}$ & $\begin{array}{c}\text { EBM solution } \\
{[14]} \\
\end{array}$ & $\begin{array}{c}R \text {. error } \\
(\%)\end{array}$ & $\begin{array}{l}\text { Present } \\
\text { solution }\end{array}$ & $\begin{array}{c}R \text {. error } \\
(\%)\end{array}$ \\
\hline 0 & 1.000000000 & 1.000000000 & 0.000000000 & 1.000000000 & 0.000000000 & 1.000000000 & 0.000000000 \\
\hline 0.5 & 0.939545503 & 0.945169971 & 0.598637105 & 0.944189348 & 0.494265045 & 0.945834709 & 0.669388122 \\
\hline 1 & 0.769687769 & 0.786692549 & 2.20930885 & 0.782987048 & 1.727879737 & 0.789206596 & 2.535940908 \\
\hline 1.5 & 0.520367974 & 0.541946378 & 4.146758655 & 0.534386712 & 2.694004762 & 0.547083274 & 5.13392471 \\
\hline 2 & 0.226896964 & 0.237770336 & 4.792206916 & 0.226137434 & 0.334746656 & 0.245694103 & 8.284438306 \\
\hline 2.5 & -0.082424976 & -0.092479615 & 12.19853434 & -0.107353589 & 30.24400395 & -0.082311253 & 0.137971529 \\
\hline 3 & -0.386261801 & -0.412588246 & 6.815699852 & -0.428861683 & 11.0287587 & -0.401399783 & 3.919098902 \\
\hline 3.5 & -0.660436345 & -0.687452426 & 4.090641165 & -0.702499667 & 6.369019864 & -0.677004442 & 2.50865918 \\
\hline 4 & -0.871990011 & -0.886930535 & 1.713382471 & -0.897723721 & 2.951147338 & -0.879268816 & 0.834734906 \\
\hline 4.5 & -0.986529936 & -0.989147789 & 0.265359712 & -0.992742682 & 0.629757473 & -0.986281489 & 0.025183929 \\
\hline 5 & -0.982752773 & -0.982895042 & 0.014476581 & -0.970950401 & 1.200950262 & -0.986449715 & 0.376182302 \\
\hline 5.5 & -0.861382758 & -0.868857967 & 0.867815025 & -0.852109657 & 1.07653664 & -0.879755271 & 2.132909305 \\
\hline 6 & -0.644890958 & -0.659541879 & 2.271844692 & -0.632155313 & 1.974852468 & -0.677756428 & 5.09628327 \\
\hline 6.5 & -0.368002797 & -0.377900390 & 2.689542873 & -0.341638967 & 7.164029789 & -0.402335838 & 9.329559797 \\
\hline 7 & -0.063204827 & -0.054818323 & 13.26877139 & -0.012988435 & 79.45024832 & -0.083329972 & 31.84115194 \\
\hline 7.5 & 0.245785975 & 0.274275125 & 11.59103972 & 0.317111883 & 29.01951912 & 0.244703077 & 0.440585758 \\
\hline 8 & 0.537446333 & 0.573291546 & 6.669542762 & 0.611815759 & 13.8375539 & 0.546227301 & 1.633831596 \\
\hline 8.5 & 0.78289188 & 0.809440784 & 3.391132885 & 0.838227903 & 7.068156461 & 0.788578403 & 0.726348445 \\
\hline 9 & 0.946701653 & 0.956826699 & 1.069507586 & 0.9716766667 & 2.638108207 & 0.945502349 & 0.12668236 \\
\hline 9.5 & 0.999764591 & 0.999286944 & 0.047775947 & 0.995531392 & 0.423419577 & 0.999999478 & 0.023494231 \\
\hline 10 & 0.931963943 & 0.932165326 & 0.021608454 & 0.908764206 & 2.489338474 & 0.946166081 & 1.523893505 \\
\hline
\end{tabular}

Thus

$$
\begin{aligned}
\left\langle\sqrt{1+u^{2}} \ddot{u} u\right\rangle & =-\omega^{2}\left\langle u^{2} \sqrt{1+u^{2}}\right\rangle, \\
\langle u \ddot{u}\rangle & =-\omega^{2}\left\langle u^{2}\right\rangle .
\end{aligned}
$$

Substituting (49) and (50) into (47), we get

$$
\omega^{2}=\frac{\left(1-\omega^{2}\right)\left\langle u^{2} \sqrt{1+u^{2}}\right\rangle}{\left\langle u^{2}\right\rangle}-\eta+\omega^{2}
$$

Using definition (11) and weighted coefficient (14), we calculate averaging operators in (51):

$$
\begin{gathered}
\left\langle u^{2}\right\rangle=\left\langle A^{2} \cos ^{2} \omega t\right\rangle=\int_{0}^{+\infty} A^{2} s^{2} \omega^{2} t e^{-s \omega t} \cos ^{2}(\omega t) d t \\
=\int_{0}^{+\infty} A^{2} s^{2} \tau e^{-s \tau} \cos ^{2}(\tau) d \tau=A^{2} \frac{s^{4}+2 s^{2}+8}{\left(s^{2}+4\right)^{2}} \\
\left\langle u^{2} \sqrt{1+u^{2}}\right\rangle=\left\langle A^{2} \cos ^{2}(\omega t) \sqrt{1+A^{2} \cos ^{2}(\omega t)}\right\rangle
\end{gathered}
$$


TABLE 3: The results of present, approximate obtained by Jouybari \& Ramzani, and numerical solution for $\theta(t)$ when $\Lambda=1$ and $A=1$, Example 2.

\begin{tabular}{|c|c|c|c|c|c|c|c|}
\hline$t$ & $\begin{array}{l}\text { Numerical } \\
\text { solution [2] }\end{array}$ & $\begin{array}{c}\text { MMA solution } \\
{[6]}\end{array}$ & $\begin{array}{c}R \text {. error } \\
(\%)\end{array}$ & $\begin{array}{c}\text { EBM solution } \\
{[14]}\end{array}$ & $\begin{array}{c}R \text {. error } \\
(\%)\end{array}$ & $\begin{array}{c}\text { Present } \\
\text { solution }\end{array}$ & $\begin{array}{c}R . \text { error } \\
(\%)\end{array}$ \\
\hline 0 & 1.000000000 & 1.000000000 & 0.000000000 & 1.000000000 & 0.000000000 & 1.000000000 & 0.000000000 \\
\hline 0.5 & 0.953968489 & 0.963119576 & 0.959265123 & 0.961786066 & 0.819479583 & 0.964200044 & 1.072525468 \\
\hline 1 & 0.825442672 & 0.855198634 & 3.604849011 & 0.850064873 & 2.982908666 & 0.859363450 & 4.109404463 \\
\hline 1.5 & 0.638888746 & 0.684197515 & 7.091808908 & 0.673375035 & 5.397855138 & 0.692996509 & 8.469043059 \\
\hline 2 & 0.422287711 & 0.462729406 & 9.576810773 & 0.445220578 & 5.430626183 & 0.477011079 & 12.95878771 \\
\hline 2.5 & 0.195637881 & 0.207129984 & 5.874170657 & 0.183038862 & 6.439969057 & 0.226871698 & 15.96511721 \\
\hline 3 & -0.032545617 & -0.063747522 & 95.87129659 & -0.093132124 & 186.1587291 & -0.039511677 & 21.40398813 \\
\hline 3.5 & -0.260619945 & -0.329922957 & 26.5915995 & -0.362185221 & 38.97064593 & -0.303066019 & 16.28657929 \\
\hline 4 & -0.485739543 & -0.571762994 & 17.70978959 & -0.603557273 & 24.25533019 & -0.544920861 & 12.18375544 \\
\hline 4.5 & -0.696309233 & -0.771429307 & 10.78832083 & -0.79880073 & 14.71925003 & -0.747759417 & 7.388984888 \\
\hline 5 & -0.869266232 & -0.914194339 & 5.16850941 & -0.93299355 & 7.331162267 & -0.897058465 & 3.197206101 \\
\hline 5.5 & -0.976272484 & -0.989527622 & 1.357729345 & -0.995879662 & 2.008371466 & -0.982128207 & 0.599804163 \\
\hline 6 & -0.996192580 & -0.991872507 & 0.43365842 & -0.982652815 & 1.35915136 & -0.996877655 & 0.068769334 \\
\hline 6.5 & -0.924854298 & -0.921056034 & 0.410687825 & -0.894323908 & 3.301102678 & -0.940250751 & 1.664743629 \\
\hline 7 & -0.776876524 & -0.782301685 & 0.698329893 & -0.737643731 & 5.050068034 & -0.816301976 & 5.074867213 \\
\hline 7.5 & -0.579081909 & -0.585844101 & 1.167743612 & -0.524587017 & 9.410567167 & -0.633906052 & 9.467424582 \\
\hline 8 & -0.358097172 & -0.346174158 & 3.329547098 & -0.271437236 & 24.20011739 & -0.406122511 & 13.41125894 \\
\hline 8.5 & -0.130576292 & -0.080970115 & 37.99018661 & 0.002457915 & 101.8823593 & -0.149260634 & 14.30913814 \\
\hline 9 & 0.097635936 & 0.190206352 & 94.8118283 & 0.276165213 & 182.8520157 & 0.118288292 & 21.15241257 \\
\hline 9.5 & 0.325420035 & 0.447353037 & 37.46942071 & 0.528765792 & 62.48716586 & 0.377367786 & 15.96329218 \\
\hline 10 & 0.548087739 & 0.671502582 & 22.51735155 & 0.740953929 & 35.1889262 & 0.609427780 & 11.19164627 \\
\hline
\end{tabular}

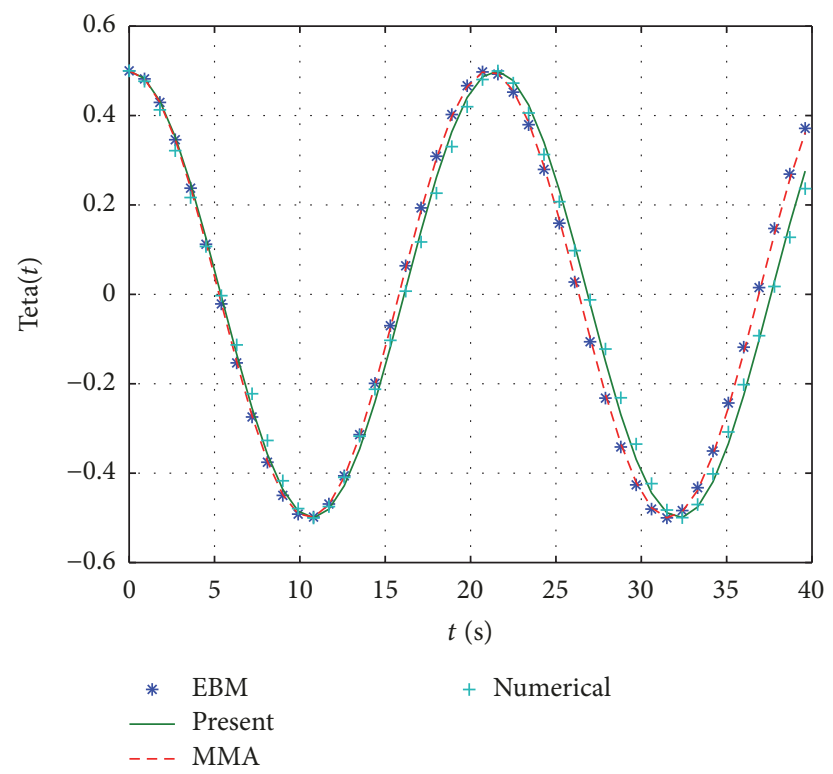

FIGURE 5: A comparison between the approximate and numerical solutions with $\Lambda=1$ and $A=0.5$, Example 2 .

$$
\begin{aligned}
& =\int_{0}^{+\infty} A^{2} s^{2} \omega^{2} t e^{-s \omega t} \cos ^{2}(\omega t) \sqrt{1+A^{2} \cos ^{2}(\omega t)} d t \\
& =\int_{0}^{+\infty} A^{2} s^{2} \tau e^{-s \tau} \cos ^{2}(\tau) \sqrt{1+A^{2} \cos ^{2}(\tau)} d \tau .
\end{aligned}
$$

With $s$ chosen equal to 2, substituting (52) into (51), we get the approximate frequency of this oscillator:

$$
\omega=\sqrt{1-\frac{\eta A^{2}}{2 I}}
$$




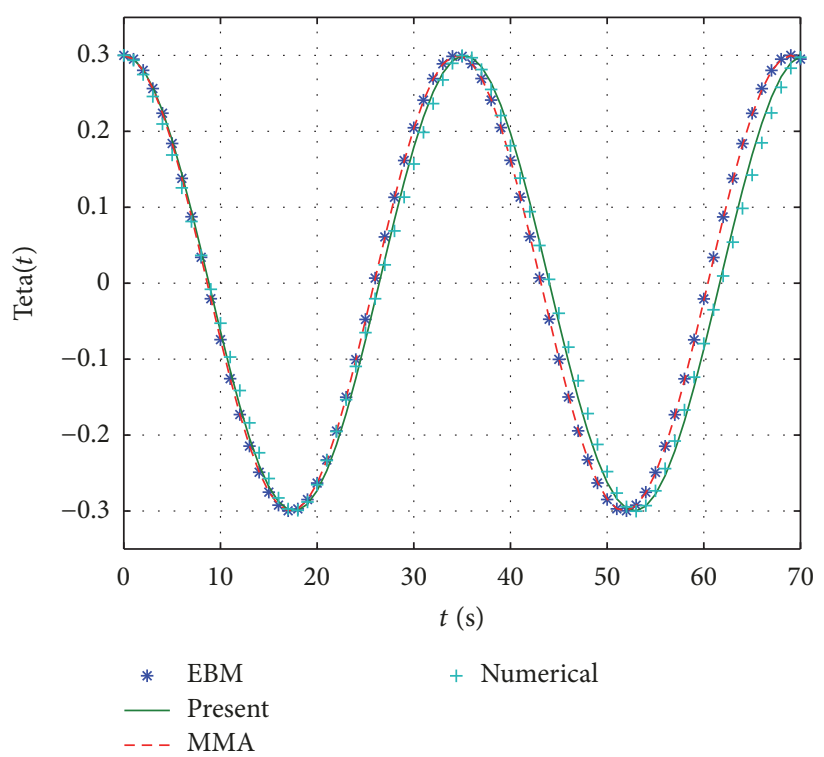

FIgURE 6: A comparison between the approximate and numerical solutions with $\Lambda=1$ and $A=0.3$, Example 2 .

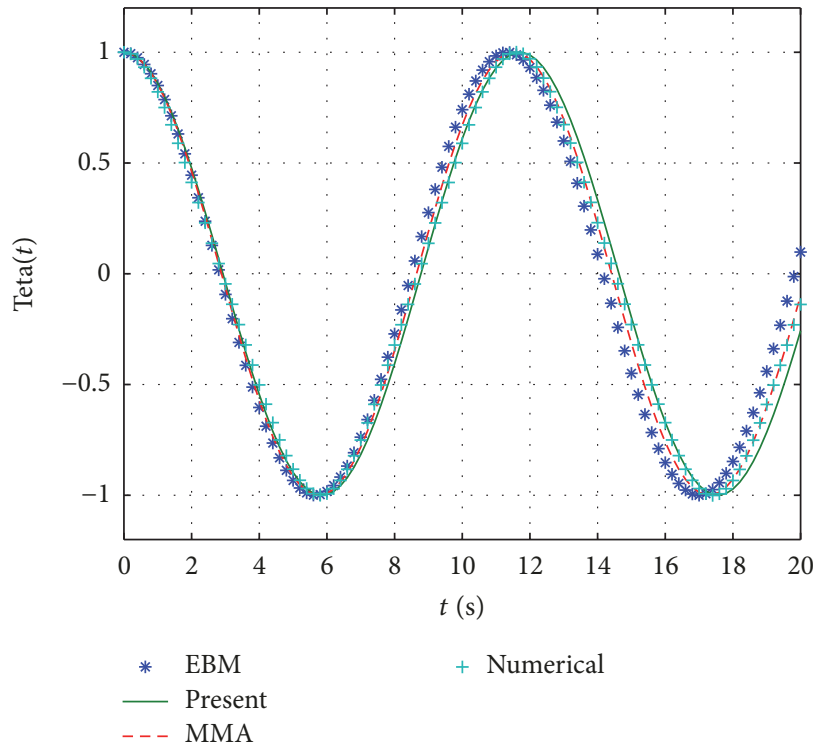

FIgURE 7: A comparison between the approximate and numerical solutions with $\Lambda=1$ and $A=1$, Example 2.

and the approximate solution of this oscillator:

$$
u(t)=A \cos \left(\sqrt{1-\frac{\eta A^{2}}{2 I}} t\right),
$$

where

$$
I=\int_{0}^{+\infty} 4 A^{2} \tau e^{-2 \tau} \cos ^{2}(\tau) \sqrt{1+A^{2} \cos ^{2}(\tau)} d \tau .
$$

Thus, the approximate period of this oscillator is

$$
T=\frac{2 \pi}{\omega}=\frac{2 \pi}{\sqrt{1-\eta A^{2} / 2 I}} .
$$

For $\eta=0.1, \eta=0.5, \eta=0.75$, and $\eta=0.95$, comparison of the approximate periods $T$ in (56), the approximate Variational Iteration Method (VIM) periods $T_{\mathrm{VIM}}$ [5] in (57), and the Energy Balance Method (EBM) periods $T_{\mathrm{EBM}}[16]$ in (58) with exact periods $T_{\text {ex }}$ in (59) is tabulated in Table 4. From Table 4, the accuracy of this proposed method can be observed.

The approximate period obtained by Variational Iteration Method is as follows [5]:

$$
T_{\mathrm{VIM}}=\frac{2 \pi \sqrt{1+A^{2}}}{\sqrt{1+A^{2}-\sqrt{1+A^{2}} \eta}} .
$$


TABLE 4: Comparison of the approximate periods with the exact periods, Example 3.

\begin{tabular}{lcccccccc}
\hline$A$ & $\eta$ & $T_{\text {ex }}[18]$ & $T_{\text {VIM }}[5]$ & $R$. error (\%) & $T_{\text {Present }}$ & $R$. error (\%) & $T_{\text {EBM }}[16]$ & $R$. error (\%) \\
\hline 0.1 & 0.1 & 6.62168 & 6.621237 & 0.00669 & 6.62174 & 0.00091 & 6.62169 & 0.00011 \\
1 & 0.1 & 6.537508 & 6.517854 & 0.30063 & 6.53806 & 0.00844 & 6.53573 & 0.02723 \\
10 & 0.1 & 6.322938 & 6.314678 & 0.13064 & 6.32097 & 0.03112 & 6.32006 \\
100 & 0.1 & 6.287182 & 6.286329 & 0.01357 & 6.28696 & 0.00353 & 6.28687 & 0.04558 \\
0.1 & 0.5 & 8.869257 & 8.863794 & 0.06159 & 8.86990 & 0.00725 & 8.86925 \\
1 & 0.5 & 7.992133 & 7.814722 & 2.21982 & 7.99408 & 0.02436 & 7.97282 & 0.00498 \\
10 & 0.5 & 6.490208 & 6.445572 & 0.68774 & 6.47925 & 0.16884 & 6.47431 & 0.24498 \\
100 & 0.5 & 6.303280 & 6.298952 & 0.06866 & 6.30214 & 0.01809 & 6.30167 \\
0.1 & 0.75 & 12.49673 & 12.47385 & 0.18309 & 12.49943 & 0.02161 & 12.4967 \\
1 & 0.75 & 9.625404 & 9.168186 & 4.75012 & 9.61936 & 0.06279 & 9.56406 & 0.02557 \\
10 & 0.75 & 6.602092 & 6.531632 & 1.06724 & 6.58442 & 0.26767 & 6.57665 \\
100 & 0.75 & 6.313404 & 6.306879 & 0.10335 & 6.31168 & 0.02731 & 6.31097 \\
0.1 & 0.95 & 27.15679 & 26.86136 & 1.08787 & 27.18975 & 0.12137 & 27.1540 \\
1 & 0.95 & 12.075277 & 10.96676 & 9.18005 & 12.00887 & 0.54994 & 0.63733 \\
10 & 0.95 & 6.696117 & 6.60302 & 1.39031 & 6.67235 & 0.35494 & 0.38536 \\
100 & 0.95 & 6.321539 & 6.313243 & 0.13123 & 6.31934 & 0.03479 & 6.6737 & 0.01043 \\
\hline
\end{tabular}

The approximate period obtained by Energy Balance Method is as follows [16]:

$$
T_{\mathrm{EBM}}=\frac{2 \pi}{(2 / A) \sqrt{A^{2} / 4-\eta\left(-\sqrt{A^{2} / 2+1}+\sqrt{A^{2}+1}\right)}} .
$$

The exact period of this oscillator is as follows [18]:

$$
T_{\mathrm{ex}}=4 \int_{0}^{\pi / 2}\left[1-\frac{2 \eta}{\sqrt{1+A^{2} \sin ^{2} \theta}+\sqrt{1+A^{2}}}\right]^{-1 / 2} d \theta
$$

Comparisons of the approximate solutions obtained by two analytical methods with the exact ones is presented in Figures 8 and 9. And the relative error versus the oscillation amplitude is illustrated in Figure 10.

3.4. Example 4. Consider the Duffing oscillator with discontinuity [3]:

$$
\ddot{u}+\alpha u+\beta u|u|=0, \quad u(0)=A, \dot{u}(0)=0 .
$$

The linearized equation of $(60)$ is

$$
\ddot{u}+\omega^{2} u=0 .
$$

The equation error between (60) and (61) is

$$
e(u)=\alpha u+\beta u|u|-\omega^{2} u .
$$

The unknown coefficient $\omega^{2}$ is determined from the mean square error criterion, as follows:

$$
\omega^{2}=\alpha+\frac{\beta\left\langle u^{2}|u|\right\rangle}{\left\langle u^{2}\right\rangle} .
$$

The periodic solution of $(61)$ is

$$
u(t)=A \cos (\omega t) .
$$

Similar to Examples 1-3, we calculate averaging operators $\left\langle u^{2}\right\rangle$ and $\left\langle u^{2}|u|\right\rangle$; then, substituting these operators into (63) with note that the parameter $s$ is chosen equal to 2 , we have the approximate frequency of this oscillator:

$$
\omega=\sqrt{\alpha+0.8324 \beta A} .
$$

Thus, the approximate solution is

$$
u(t)=A \cos (\sqrt{\alpha+0.8324 \varepsilon A} t) .
$$

Accuracy of the approach for this example is shown in Figures 11 and 12. We performed a comparison between the results obtained by this method, the ones obtained by $\mathrm{He}$ [3] using the Homotopy Perturbation Method, and outcomes achieved using the Runge-Kutta 4th-Order Method for different values of $A, \alpha$, and $\beta$.

3.5. Example 5. Consider the nonlinear oscillator equation of the form $[23,24,34]$

$$
\ddot{u}+u^{1 /(2 n+1)}=0, \quad u(0)=A, \dot{u}(0)=0,
$$

where $n$ is a positive integer

The linearized equation of (67) is

$$
\ddot{u}+\omega^{2} u=0 .
$$

The equation error between (67) and (68) is

$$
e(u)=u^{1 /(2 n+1)}-\omega^{2} u .
$$

The unknown coefficient $\omega^{2}$ is determined from the mean square error criterion, as follows:

$$
\omega^{2}=\frac{\left\langle u u^{1 /(2 n+1)}\right\rangle}{\left\langle u^{2}\right\rangle} .
$$




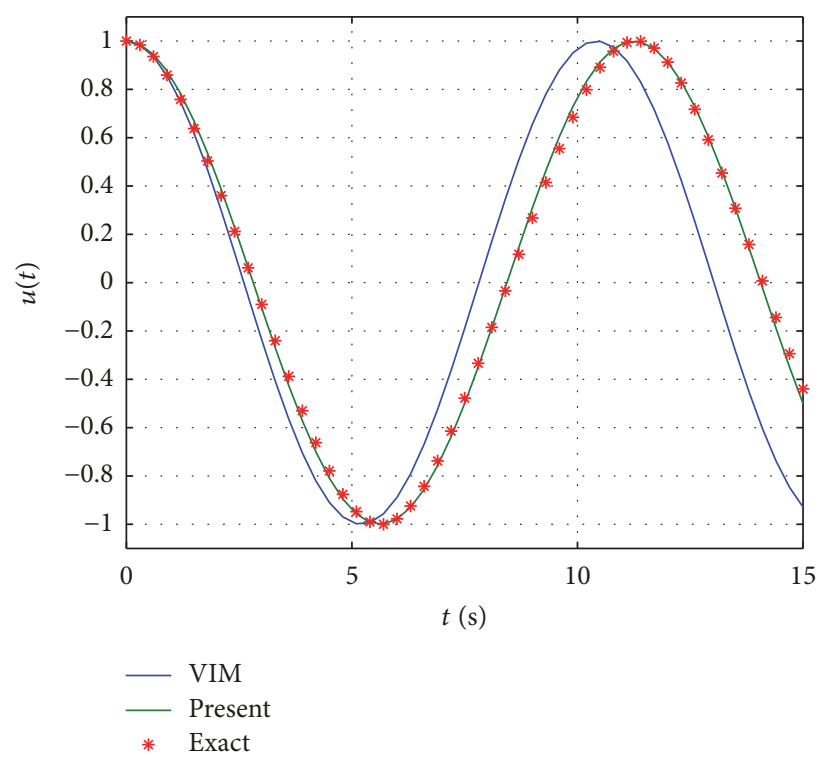

FIGURE 8: Comparison of time history diagram of displacement between the present, VIM, and exact solutions at $\eta=0.9, A=1$, Example 3 .

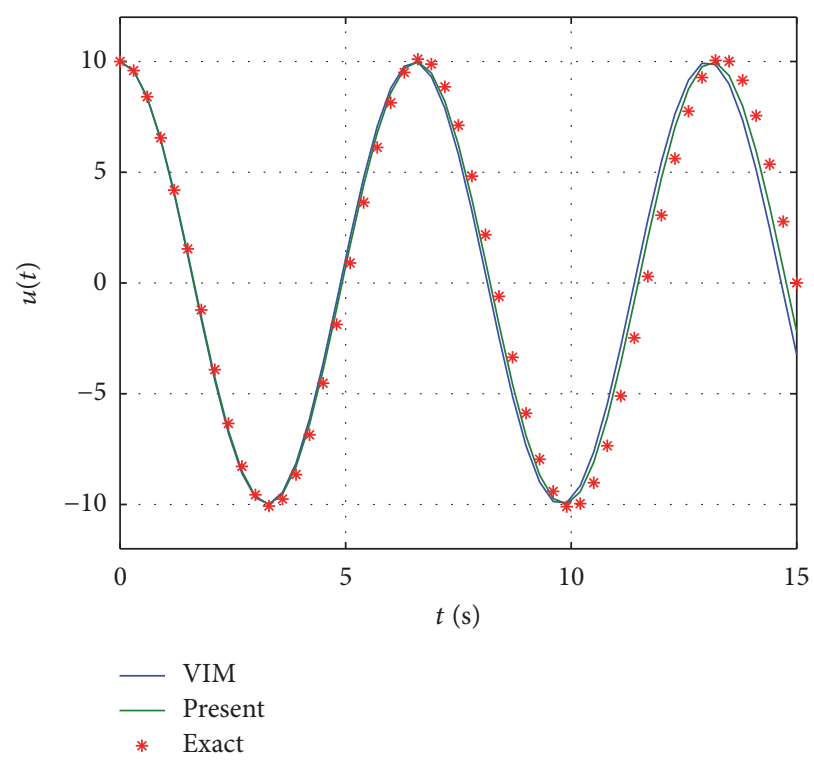

FIGURE 9: Comparison of time history diagram of displacement between the present, VIM, and exact solutions at $\eta=0.7, A=10$, Example 3.

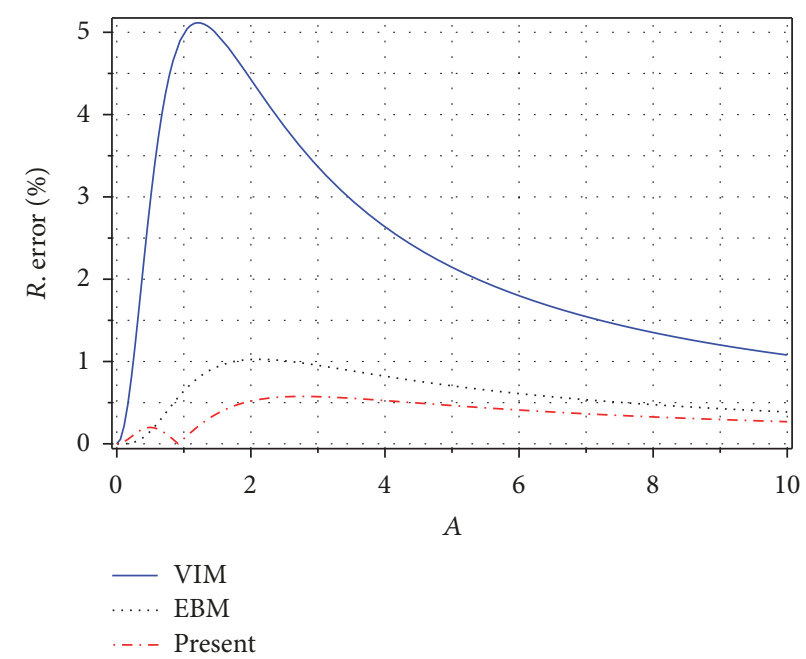

FIGURE 10: Comparison of relative error between the present, VIM, and EBM solutions with $\eta=0.75$, Example 3. 


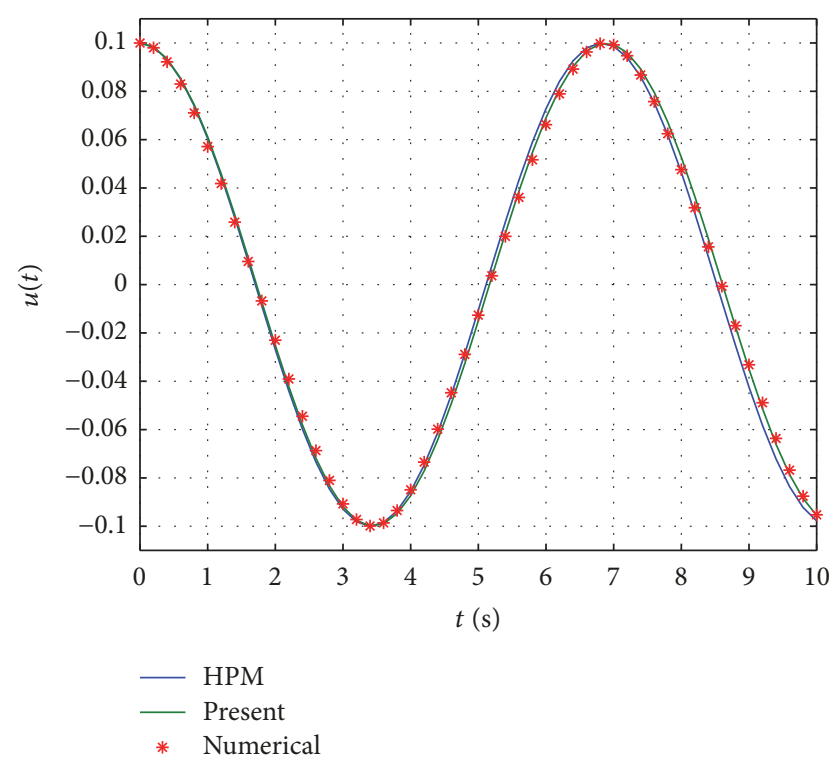

FIGURE 11: Comparison of time history diagram of displacement between the present, HPM, and numerical solutions at $\alpha=0, \beta=$ 10, $A=0.1$, Example 4 .

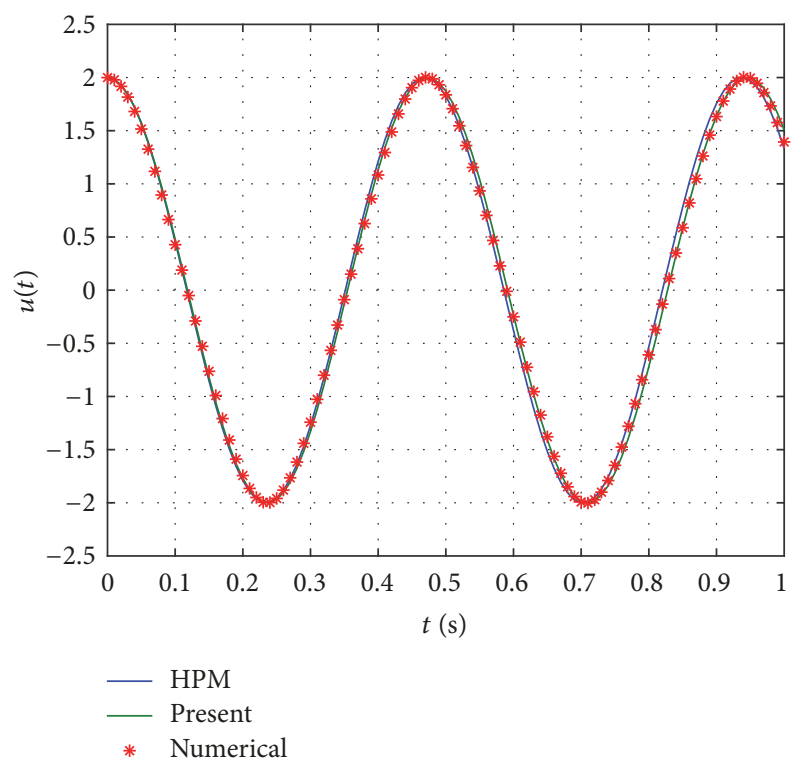

FIGURE 12: Comparison of time history diagram of displacement between the Present, HPM, and numerical solutions at $\alpha=10, \beta=$ 100, $A=2$, Example 4.

The periodic solution of (68) is

$$
u(t)=A \cos (\omega t)
$$

Using definition (11) and weighted coefficient (14), we calculate averaging operators in (70):

$$
\begin{aligned}
& \left\langle u^{2}\right\rangle=\left\langle A^{2} \cos ^{2} \omega t\right\rangle=\int_{0}^{+\infty} A^{2} s^{2} \omega^{2} t e^{-s \omega t} \cos ^{2}(\omega t) d t \\
& =\int_{0}^{+\infty} A^{2} s^{2} \tau e^{-s \tau} \cos ^{2}(\tau) d \tau=A^{2} \frac{s^{4}+2 s^{2}+8}{\left(s^{2}+4\right)^{2}} \\
& \left\langle u u^{1 /(2 n+1)}\right\rangle=\left\langle A \cos (\omega t) A^{1 /(2 n+1)}(\cos (\omega t))^{1 /(2 n+1)}\right\rangle \\
& =\int_{0}^{+\infty} A A^{1 /(2 n+1)} s^{2} \omega^{2} t e^{-s \omega t} \cos (\omega t) \\
& \cdot(\cos (\omega t))^{1 /(2 n+1)} d t=\int_{0}^{+\infty} A A^{1 /(2 n+1)} s^{2} \tau e^{-s \tau} \\
& \cdot \cos (\tau)(\cos (\tau))^{1 /(2 n+1)} d \tau .
\end{aligned}
$$

With $s$ chosen equal to 2, from (72) and (73), we have

$$
\begin{aligned}
& \left\langle u^{2}\right\rangle=\frac{A^{2}}{2} \\
& \left\langle u u^{1 /(2 n+1)}\right\rangle=\int_{0}^{+\infty} A^{(2 n+2) /(2 n+1)} 4 \tau e^{-2 \tau} \cos (\tau) \\
& \cdot(\cos (\tau))^{1 /(2 n+1)} d \tau .
\end{aligned}
$$

Substituting (71) and (72) into (70), we get the approximate frequency of this oscillator:

$$
\omega=A^{-n /(2 n+1)} \sqrt{2 I_{n}}
$$

where

$$
I_{n}=\int_{0}^{+\infty} 4 \tau e^{-2 \tau} \cos (\tau)(\cos (\tau))^{1 /(2 n+1)} d \tau .
$$

For $n=0$ (the harmonic oscillator case) it follows from (77) and (76) that $\omega$ is equal to the well-known value 1 . And, for $n \rightarrow \infty$, $\omega$ is equal to $0.97979 A^{-1 / 2}$. For other values of $n$, the integral in (77) can be calculated numerically by using the formula manipulation package Maple. For some values of $n$ approximations of the frequency are given in Table 5. Table 5 presents the comparison of the approximate frequencies $\omega_{\text {present }}$ in (76), the approximate frequencies $\omega_{\text {app }}$ obtained by van Horssen [34], and the approximate frequencies $\omega_{\text {HBM }}$ obtained by Mickens [23]. As can be seen from this table the fractional errors of the approximations obtained in [34] for $n \geq 1$ range from approximately 2 to approximately 11 percent, while the fractional errors of the approximations obtained in this paper range from 2 to 10 percent. And, from Table 5, when $n$ increases, the relative error of the approximate frequencies $\omega_{\text {app }}$ obtained by van Horssen increases, while the relative error of the approximate frequencies $\omega_{\text {app }}$ in this paper decreases.

Comparison of the approximate solutions obtained by three methods is present in Figures 13 and 14.

\section{Conclusions}

In this paper, the Equivalent Linearization Method with a weighted averaging is applied to analyze five undamped 
TABLE 5: Comparison of the approximate frequencies, Example 5.

\begin{tabular}{|c|c|c|c|c|c|}
\hline$n$ & $\omega_{\mathrm{HBM}}[23]$ & $\omega_{\text {app }}[34]$ & $R$. error $(\%)$ & $\omega_{\text {present }}$ & R. error (\%) \\
\hline 0 & 1.00000 & 1.000000 & 0.00000 & 1.000000 & 0.00000 \\
\hline 1 & $A^{-1 / 3} 1.04912$ & $A^{-1 / 3} 1.07045$ & 2.03313 & $A^{-1 / 3} 0.94307$ & 10.10847 \\
\hline 2 & $A^{-2 / 5} 1.04812$ & $A^{-2 / 5} 1.08613$ & 3.62649 & $A^{-2 / 5} 0.95714$ & 8.680304 \\
\hline 3 & $A^{-3 / 7} 1.04405$ & $A^{-3 / 7} 1.09302$ & 4.69039 & $A^{-3 / 7} 0.96342$ & 7.72281 \\
\hline 4 & $A^{-4 / 9} 1.04017$ & $A^{-4 / 9} 1.09689$ & 5.45295 & $A^{-4 / 9} 0.96697$ & 7.037311 \\
\hline 5 & $A^{-5 / 11} 1.03684$ & $A^{-5 / 11} 1.09938$ & 6.03179 & $A^{-5 / 11} 0.96926$ & 6.517881 \\
\hline 6 & $A^{-6 / 13} 1.03403$ & $A^{-6 / 13} 1.10110$ & 6.48627 & $A^{-6 / 13} 0.97085$ & 6.110074 \\
\hline 7 & $A^{-7 / 15} 1.03164$ & $A^{-7 / 15} 1.10238$ & 6.85704 & $A^{-7 / 15} 0.97202$ & 5.779148 \\
\hline 8 & $A^{-8 / 17} 1.02960$ & $A^{-8 / 17} 1.10335$ & 7.16298 & $A^{-8 / 17} 0.97293$ & 5.504079 \\
\hline 9 & $A^{-9 / 19} 1.02783$ & $A^{-9 / 19} 1.10412$ & 7.42243 & $A^{-9 / 19} 0.97364$ & 5.272273 \\
\hline 10 & $A^{-10 / 21} 1.02628$ & $A^{-10 / 21} 1.10474$ & 7.64509 & $A^{-10 / 21} 0.97422$ & 5.07269 \\
\hline 50 & $A^{-50 / 101} 1.00919$ & $A^{-50 / 101} 1.10947$ & 9.93668 & $A^{-50 / 101} 0.97863$ & 3.028171 \\
\hline 500 & $A^{-500 / 1001} 1.00149$ & $A^{-500 / 1001} 1.11059$ & 10.8938 & $A^{-500 / 1001} 0.97968$ & 2.17776 \\
\hline$\infty$ & $A^{-1 / 2} 1.00000$ & $A^{-1 / 2}(\pi / 2 \sqrt{2})$ & 11.07200 & $A^{-1 / 2} 0.97979$ & 2.02100 \\
\hline
\end{tabular}

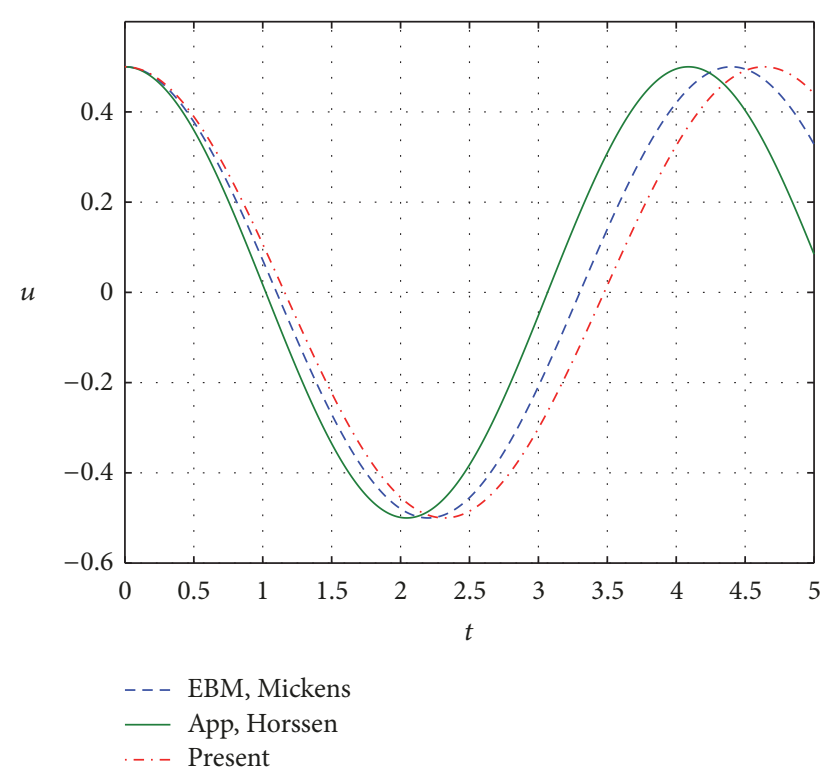

FIGURE 13: Comparison of time history diagram of displacement between the present, HBM [23], and App [34] solutions with $n=10$, Example 5.

nonlinear oscillation systems. This method is proposed by Anh in 2015. Instead of using the classical averaging process in (9) and (10), a special form of weighted coefficient $h(t)$ is introduced in (14), whereby a new weighted average value is computed by (15). The proposed weighted function is a combination of two basic coefficient functions (the optimistic weighted coefficient $t$ and the pessimistic weighted coefficient $\left.e^{-s \omega t}\right)$. The frequency-amplitude relationship was analytically obtained by this method. The frequency depends not only on the initial amplitude of the oscillator but also on the parameter $s$ in the expression of weighted coefficient $h(t)$. Accuracy of this method is investigated by five nonlinear oscillation systems. The results show that this method is useful in obtaining analytical solutions for oscillators and vibration problems with nonlinearities. The approximate solutions obtained by this method are valid not only for small parameters but also for very large parameters. And the results indicate that the solution procedure is easy and provides a remarkable accuracy. In this paper, $s$ is chosen equal to 2 for comparison and convenient calculating purposes. However, the value of the parameter $s$ in the expression of the weighted coefficient $h(t)$ should be chosen to give better results and the best solution still requires further investigation.

\section{Conflicts of Interest}

The authors declare that no conflicts of interest took place during the preparation of the manuscript. 


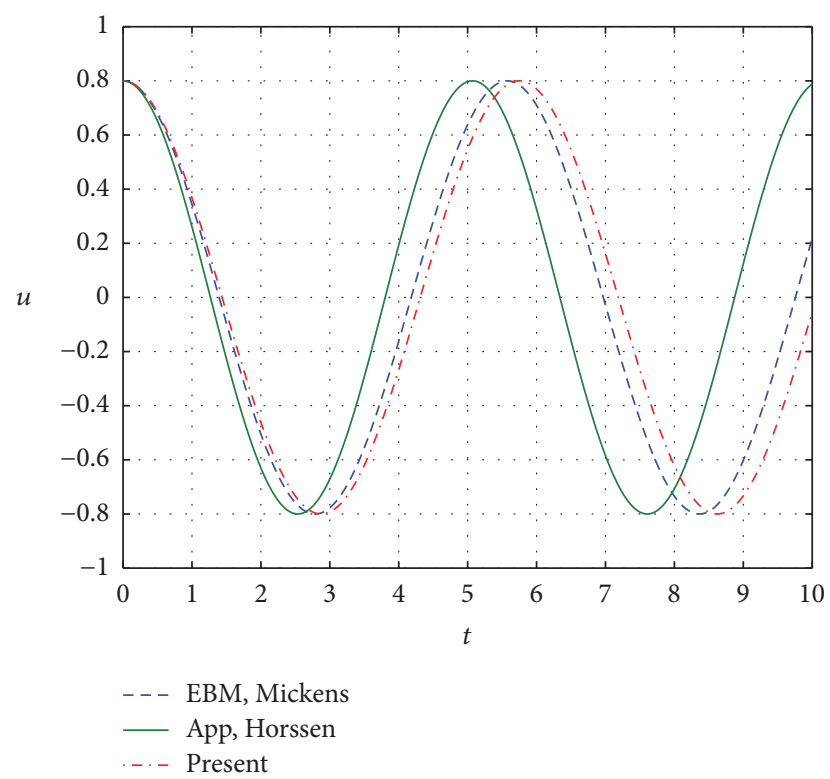

FIGURE 14: Comparison of time history diagram of displacement between the Present, HBM [23] and App [34] solutions with $n=50$, Example 5.

\section{Acknowledgments}

The research is supported by Thai Nguyen University of Technology grant for a scientific project (no. “T2018-B27”).

\section{References}

[1] T. Öziş and A. Yildirim, "A note on He's homotopy perturbation method for van der Pol oscillator with very strong nonlinearity," Chaos, Solitons \& Fractals, vol. 34, no. 3, pp. 989-991, 2007.

[2] S. Ghafoori, M. Motevalli, M. G. Nejad, F. Shakeri, D. D. Ganji, and M. Jalaal, "Efficiency of differential transformation method for nonlinear oscillation: Comparison with HPM and VIM," Current Applied Physics, vol. 11, no. 4, pp. 965-971, 2011.

[3] J.-H. He, "The homotopy perturbation method nonlinear oscillators with discontinuities," Applied Mathematics and Computation, vol. 151, no. 1, pp. 287-292, 2004.

[4] J.-H. He, "Homotopy perturbation technique," Computer Methods Applied Mechanics and Engineering, vol. 178, no. 3-4, pp. 257-262, 1999.

[5] M. Bayat, I. Pakar, and G. Domairry, "Recent developments of some asymptotic methods and their applications for nonlinear vibration equations in engineering problems: A review," Latin American Journal of Solids and Structures, vol. 9, no. 2, pp. 145234, 2012.

[6] A. K. Jouybari and M. Ramzani, "Analytical methods for solving nonlinear motion of simple pendulum attached to a rotating rigid frame," International Journal of Mechatronics, Electrical and Computer Technology, vol. 4, pp. 11-22, 2014.

[7] S. Durmaz, S. A. Demirbağ, and M. O. Kaya, "Approximate solutions for nonlinear oscillation of a mass attached to a stretched elastic wire," Computers and Mathematics with Applications, vol. 61, no. 3, pp. 578-585, 2011.

[8] M. K. Yazdi, H. Ahmadian, A. Mirzabeigy, and A. Yildirim, "Dynamic analysis of vibrating systems with nonlinearities,"
Communications in Theoretical Physics, vol. 57, no. 2, pp. 183187, 2012.

[9] J. H. He, "Max-min approach to nonlinear oscillators," International Journal of Nonlinear Sciences and Numerical Simulation, vol. 9, no. 2, pp. 207-210, 2008.

[10] S. S. Ganji, D. D. Ganji, A. G. Davodi, and S. Karimpour, "Analytical solution to nonlinear oscillation system of the motion of a rigid rod rocking back using max-min approach," Applied Mathematical Modelling: Simulation and Computation for Engineering and Environmental Systems, vol. 34, no. 9, pp. 2676$2684,2010$.

[11] H. Ebrahimi Khah and D. D. Ganji, "A study on the motion of a rigid rod rocking back and cubic-quintic duffing oscillators by using He's energy balance method," International Journal of Nonlinear Science, vol. 10, no. 4, pp. 447-451, 2010.

[12] D. Younesian, H. Askari, Z. Saadatnia, and M. KalamiYazdi, "Frequency analysis of strongly nonlinear generalized Duffing oscillators using HE's frequency-amplitude formulation and HE's energy balance method," Computers \& Mathematics with Applications. An International Journal, vol. 59, no. 9, pp. 32223228,2010

[13] S. S. Ganji, D. D. Ganji, Z. Z. Ganji, and S. Karimpour, "Periodic solution for strongly nonlinear vibration systems by He's energy balance method," Acta Applicandae Mathematicae, vol. 106, no. 1, pp. 79-92, 2009.

[14] D. Younesian, H. Askari, Z. Saadatnia, and A. Yildirim, "Periodic solutions for the generalized nonlinear oscillators containing fraction order elastic force," International Journal of Nonlinear Sciences and Numerical Simulation, vol. 11, no. 12, pp. 1027-1032, 2010.

[15] D. Younesian, H. Askari, Z. Saadatnia, and M. KalamiYazdi, "Frequency analysis of strongly nonlinear generalized Duffing oscillators using HE's frequency-amplitude formulation and HE's energy balance method," Computers \& Mathematics with Applications, vol. 59, no. 9, pp. 3222-3228, 2010.

[16] D. Younesian, H. Askari, Z. Saadatnia, and M. KalamiYazdi, "Analytical approximate solutions for the generalized nonlinear oscillator," Applicable Analysis: An International Journal, vol. 91, no. 5, pp. 965-977, 2012.

[17] G.-h. Chen, Z.-L. Tao, and J.-Z. Min, "Notes on a conservative nonlinear oscillator," Computers \& Mathematics with Applications, vol. 61, no. 8, pp. 2120-2122, 2011.

[18] L. Xu, "Application of He's parameter-expansion method to an oscillation of a mass attached to a stretched elastic wire," Physics Letters A, vol. 368, no. 3-4, pp. 259-262, 2007.

[19] M. O. Kaya and S. Altay Demirbağ, "Application of parameter expansion method to the generalized nonlinear discontinuity equation," Chaos, Solitons \& Fractals, vol. 42, no. 4, pp. 19671973, 2009.

[20] M. T. Darvishi, A. Karami, and B.-C. Shin, "Application of He’s parameter-expansion method for oscillators with smooth odd nonlinearities," Physics Letters A, vol. 372, no. 33, pp. 5381-5384, 2008.

[21] J.-H. He, "Comparison of homotopy perturbation method and homotopy analysis method," Applied Mathematics and Computation, vol. 156, no. 2, pp. 527-539, 2004.

[22] J.-H. He, "Modified Lindstedt-Poincare methods for some strongly non-linear oscillations," International Journal of NonLinear Mechanics, vol. 37, no. 2, pp. 309-314, 2002.

[23] R. E. Mickens, "Oscillations in an $x^{4 / 3}$ potential," Journal of Sound and Vibration, vol. 246, no. 2, pp. 375-378, 2001. 
[24] K. Cooper and R. E. Mickens, "Generalized harmonic balance/ numerical method for determining analytical approximations to the periodic solutions of the $x^{4 / 3}$ potential," Journal of Sound and Vibration, vol. 250, no. 5, pp. 951-954, 2002.

[25] C. W. Lim, B. S. Wu, and W. P. Sun, "Higher accuracy analytical approximations to the Duffing-harmonic oscillator," Journal of Sound and Vibration, vol. 296, no. 4-5, pp. 1039-1045, 2006.

[26] N. Krylov and N. Bogoliuboff, Introduction to nonlinear mechanics, Princenton University Press, New York, NY, USA, 1943.

[27] T. K. Caughey, "Equivalent linearization techniques," The Journal of the Acoustical Society of America, vol. 35, pp. 1706-1711, 1959.

[28] R. N. Iyengar, "Higher order linearization in nonlinear random vibration," International Journal of Non-Linear Mechanics, vol. 23, no. 5-6, pp. 385-391, 1988.

[29] N. D. Anh and W. Schiehlen, "New criterion for Gaussian equivalent linearization," European Journal of Mechanics. A Solids, vol. 16, no. 6, pp. 1025-1039, 1997.

[30] N. D. Anh and N. N. Linh, "Some extensions of Gaussian equivalent linearization," in Proceedings of the International Conference on Nonlinear Stochastic Dynamics, vol. 36, Hanoi, Vietnam, 1995.

[31] N. D. Anh, "Short Communication Dual approach to averaged values of functions: a form for weighting coefficient," Vietnam Journal of Mechanics, vol. 37, no. 2, pp. 145-150, 2015.

[32] I. Elishakoff, L. Andriamasy, and M. Dolley, "Application and extension of the stochastic linearization by Anh and Di Paola," Acta Mechanica, vol. 204, no. 1-2, pp. 89-98, 2009.

[33] N. D. Anh, N. Q. Hai, and D. V. Hieu, "The equivalent linearization method with a weighted averaging for analyzing of nonlinear vibrating systems," Latin American Journal of Solids and Structures, vol. 14, no. 9, pp. 1723-1740, 2017.

[34] W. T. van Horssen, "On the periods of the periodicsolutions of the non-linear oscillator equation," Journal of Sound and Vibration 260, pp. 961-964, 2003. 


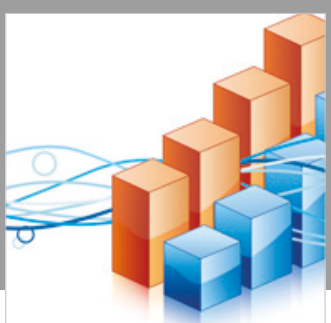

Advances in

Operations Research

\section{-n-m}
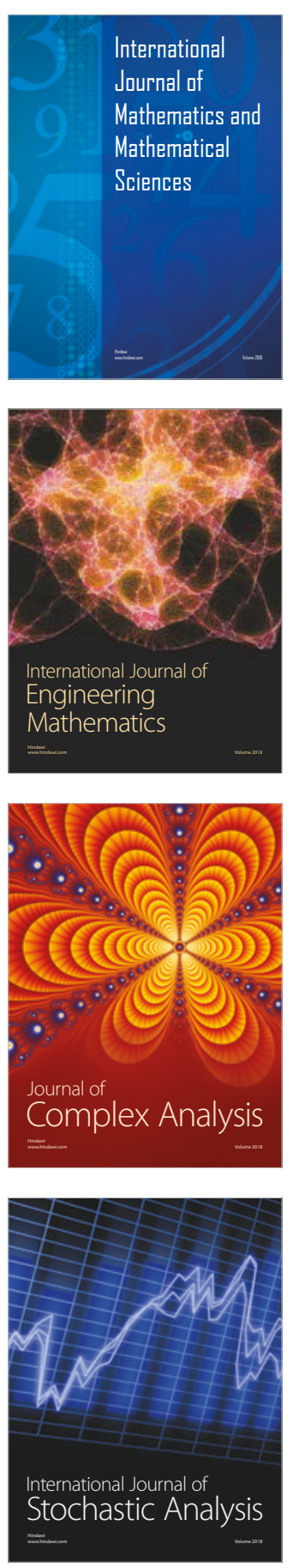
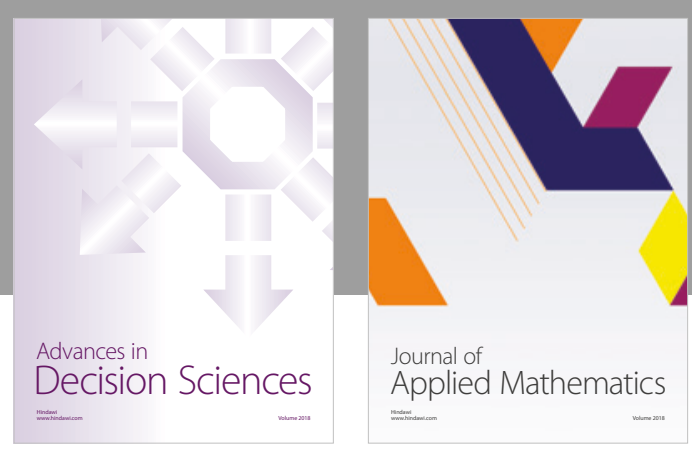

Journal of

Applied Mathematics
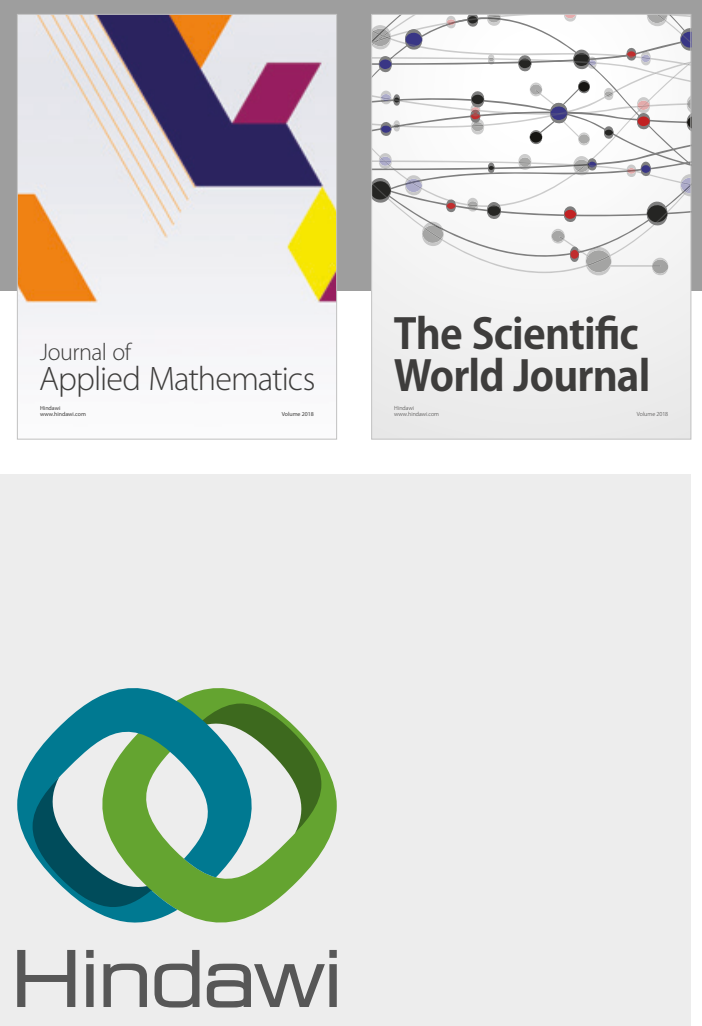

Submit your manuscripts at

www.hindawi.com

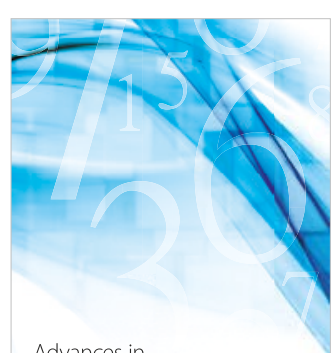

Advances in
Numerical Analysis
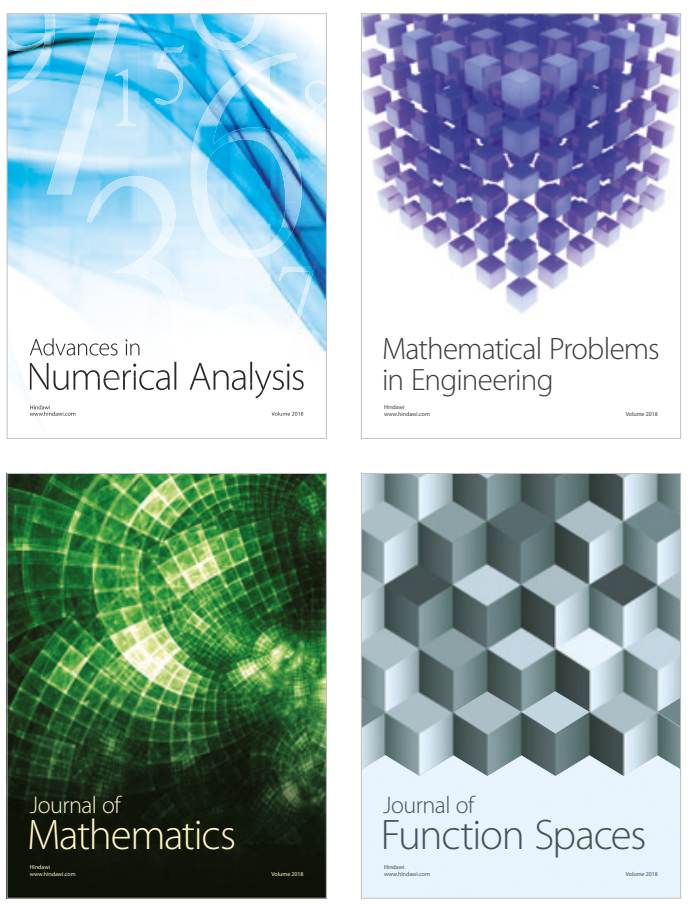

Mathematical Problems in Engineering

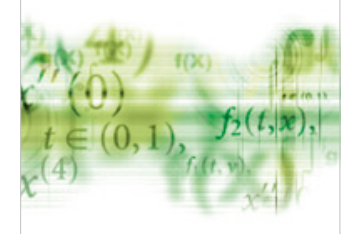

International Journal of

Differential Equations

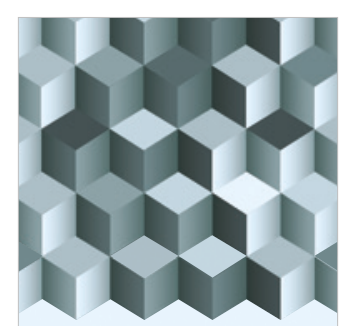

Journal of

Function Spaces

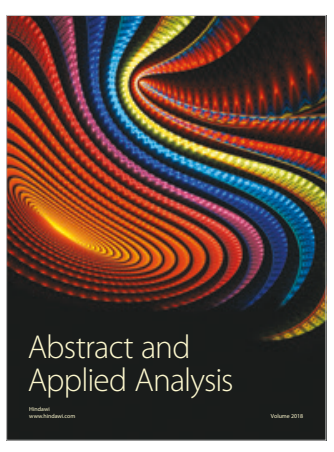

The Scientific

World Journal

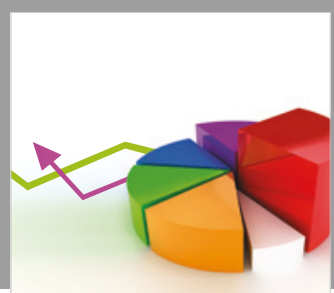

Journal of

Probability and Statistics
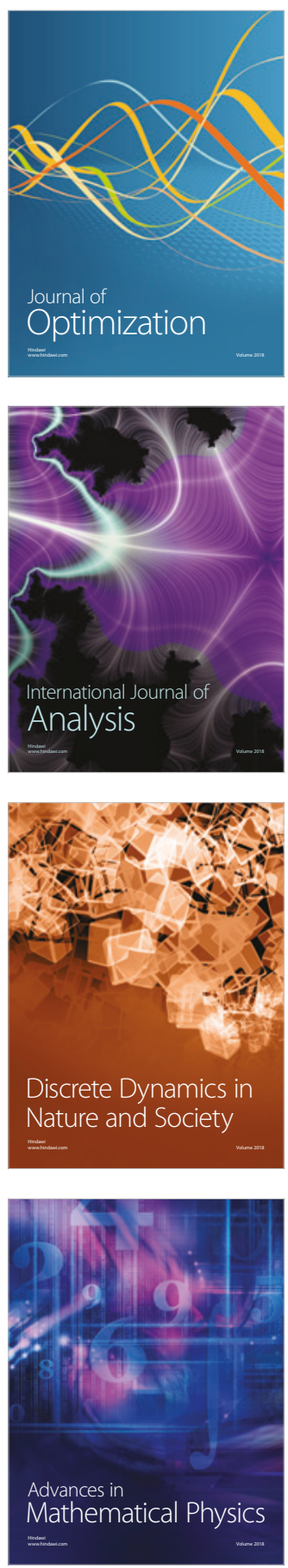